\section{A FORTRAN IV program for computing all possible multiple regressions}

\section{W. JAY HANSCHE and WILLIAM P. DUNLAP \\ Tulane University, New Orleans, Louisiana 70118}

In attempting to find the "best" regression equation when there are many predictor variables, several factors must be considered. First, one wants an equation, or model, with as few predictors as possible, since equations with many predictors are not only clumsy to use, but also tend to capitalize on predicting random variability within a particular sample at the expense of any true relation that may actually exist. Second, one wants a procedure that will not overlook certain complicated interrelations among predictors that sometimes occur. For example, a particular predictor that, at first, appears to offer very little in terms of prediction of the criterion may account for a great deal of criterion variance, when taken in conjunction with other predictors-the so-called "suppressor" variable relationship (for a cogent discussion see Darlington, 1968). A number of multiple regression procedures have been suggested, e.g., stepwise regression and backward elimination, but only one method guarantees the user the "best" possible model, and that is to examine all possible regression equations. Draper and Smith (1966) argue that, although computing all regressions is the surest approach, the enormity of the task makes alternate procedures preferable. Even granted all regressions were calculated, they argue, merely examining all of the multiple Rs becomes a difficult proposition. Using the present program, however, we have found that computing all regressions of a given order, that is, those with constant numbers of predictors, does not unduly tax the abilities of the high-speed computer. Furthermore, since one usually does not want a final model with very many predictors, one can examine all possible models from order to order, stopping when tests of significance indicate that further gains are not being made by adding variables.

Perhaps the most important advantage of examining all possible regressions is that this technique alone will provide the user with alternate models that may be almost as good as, and not differ significantly from, the "best" model. As soon as one begins to examine and compare the predictors in terms of cost as well as significance, the present approach offers an extremely important advantage over those techniques whose purpose is to zoom in on the "best" regression equation, ignoring all others. Last, the computer can do most of the preliminary editing of the regression equations, reporting only those that surpass a fixed criterion or that make an improvement over the best model of a previous

Preparation and testing of this program was supported by the Tulane University Computer Center. order; such optional editing procedures are included in the present program.

With $n$ total predictors there are $n ! /(k !(n-k) !)$ possible equations of order $k$, i.e., all possible sets of $k$ predictors chosen from $\mathrm{n}$ possible predictors. With only moderate sizes of $\mathrm{k}$, this number becomes extremely large when there are many possible predictors. A very fast iterative procedure which approximates $R$ to any preset limit of accuracy (Greenberger \& Ward, Note 1) is used in the present program. The FORTRAN code for the iterative procedure comes from Veldman's (1967) program REGRAN, which is also designed to calculate many possible regression equations. In order to modify the REGRAN program to find all possible models of a given order, a subroutine COMB was written which produces a new combination of $\mathrm{k}$ integers, given the preceding combination selected from the integers 1 to $\mathrm{N}$. The subroutine FPRB, described by Dunlap and Duffy (1975), is used to calculate the probabilities of $F$ ratios associated with each model.

Input. The correlation matrix is computed from raw data. Options for editing of regression equations are: (a) no editing, (b) fixed criterion for $R$, (c) variable criterion for $\mathrm{R}$ such that only those models whose Rs are larger than the best model of the previous order are output. A separate control card is read for each order of model such that, for example, all regression equations for models with two, three, and four predictors could be computed, each order with its own editing option.

Output. For each regression to be output, the following is printed: $R, R$ squared, $F$, probability associated with $F$, and identification of the variables included in that particular solution. At the end of all regressions of a given order, the average error mean square is printed.

Time. In order to obtain estimates of the time required to calculate all possible regressions of a given order with the present program, 10 sets of randomly generated data were used, where variable 10 was the criterion and the nine other variables were possible predictors. For two-predictor models, regressions were calculated at a rate of $12,960 / \mathrm{min}$. Models with three predictors produced 5,400 regressions/min; four predictors, 2,835/min; five predictors, $1,738 / \mathrm{min}$; and six predictors, $1,069 / \mathrm{min}$. Since the computer used was a second generation IBM 7044, one might anticipate considerably faster times with later model machines.

Computer and Language. The program was written in standard FORTRAN IV and runs on an IBM 7044; however, it should also run on any computer with a standard FORTRAN IV compiler. Data storage is not excessive and could be easily modified by the user for smaller computers.

Availability. A listing of the program and instruc- 
tions for use are available free of charge from either author at the Department of Psychology, Tulane University, New Orleans, Louisiana 70118.

\section{REFERENCE NOTE}

1. Greenberger. M. H., \& Ward, J. H., Jr. An iterative technique for multiple correlation analysis. IBM Technical Newsletter, 1956, 12, 85-97.

\section{REFERENCES}

Darlington. R. B. Multiple regression in psychological research and practice. Psychological Bulletin, 1968, 69, 161-182. Draper, N. R., \& SMith. H. Applied regression analysis. New York: Wiley, $1 \% 66$.

Dunlap, W. P., \& Duffy, J. A. FORTRAN IV functions for calculating exact probabilities associated with $z, \chi 2$, $t$, and $\mathrm{F}$ values. Behavior Research Methods \& Instrumentation, 1975, 7, 59-60.

VELDMAN, D. J. FORTRAN programming for the behavioral sciences. New York: Holt, Rinehart, \& Winston, 1967. 